\title{
Native or surgical bypass? seeking for a new predictor for angiogenesis
}

\begin{abstract}
Alat I*
The amputations following peripheral arterial occlusions that develop as a consequence of diseases such as diabetes, atherosclerosis is a stalemate of the today's people, despite the current technologies in medicine and surgery. Many methods including above and below knee bypass surgeries, peripheral angioplasty procedures, atherectomies and hybrid procedures have been developed along with technological possibilities for avoiding amputation in non-traumatic cases. Limb salvage is considered the main purpose of these applications. However, according to the previous literature, even if new techniques such as angioplasties were added to the treatment protocols, it couldn't be enough to lower the amputation rate, unfortunately [1]. On the other hand, in order to induce angiogenesis, some new therapeutic interventions [2] or some special stem cell groups [3,4] to cure critical limb ischemia have being reported recently in regard to their potential useful and hopeful effects.
\end{abstract}

Associate Professor, Department of Cardiovascular Surgery, Afyonkarahisar State Hospital, Afyonkarahisar, Turkey

It is precisely at this point that the question of how the clinician/ surgeon should make a decision comes to mind. The question of what surgeons need to guide them is gaining in importance. So, seeking for a new predictor for this purpose is gaining in importance.

Because, both applications -surgical bypass operations and induced angiogenesis- have some potential risks and benefits. For example, one of the most important problems of a surgically bypassed graft is the affected patency rate due to subsequent occlusions as a result of atherothrombogenic events [5]. Several different factors can be mentioned related to this important problem. For example, the types of grafts [5] or surgical application faults and experiences [6] can be mentioned. As an important example, if a surgeon places a very long graft within the excessive quantities of unnecessary folds, this will be really a serious surgical error. Because folds are related to lower patency rates for bypassed vascular grafts.

However, as seen in the (Figure 1), in this elderly patient, a long and curled native collateral artery, possibly developed as a result of angiogenesis induced with chronic ischemia, exhibits a good visualization as much as a surgically bypassed graft. Despite of its excessive folds, it seems that it is patent and works as a native bypass graft. Besides, when compared to the diameter of this native collateral artery to his native arterial construction, it can be seen that it is enough as much as his native popliteal artery, seen in the (Figure 2).

These events bring new thinking or understanding about the hemodynamic principles of native bypassed grafts, collateral arteries developed due to angiogenesis. Is a native bypass graft better than a surgically bypassed graft? Does it have a longer patency rate? These questions need further investigations. But, there is another important question at this point: When should a surgeon make a decision for

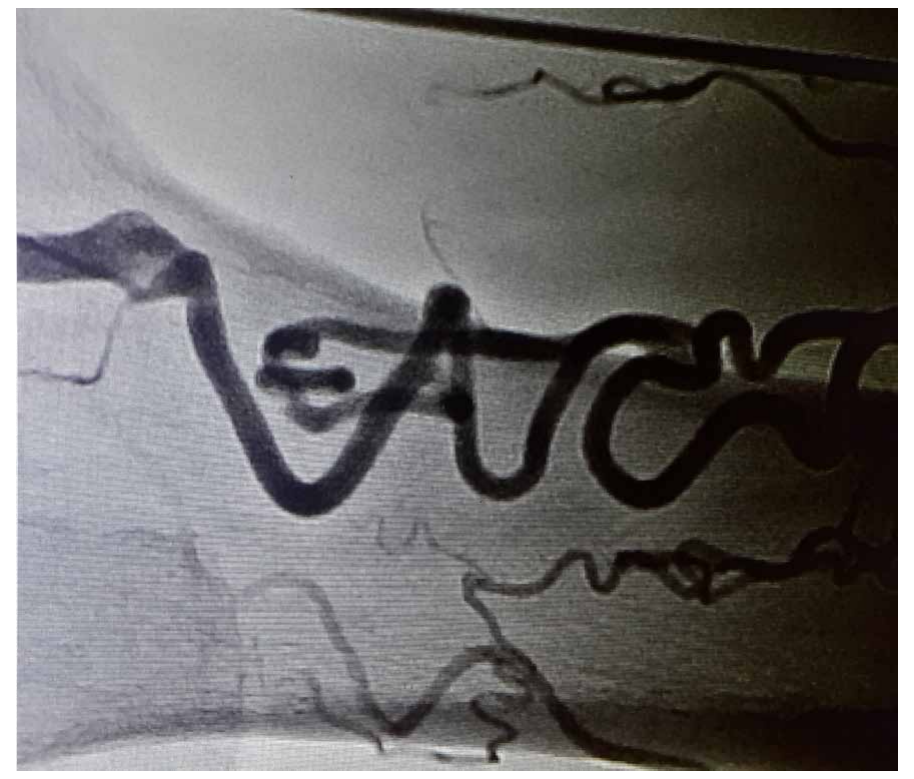

Figure 1. An angiographic view; a collateral artery is seen as a native bypass graft, developed as a result of angiogenesis due to hypoxic conditions in the limb. Note the course of this collateral artery with the excessive folds

surgical bypass operation? If a patient has a native bypass graft as seen in our case, should surgeon plan a surgery or not? How long does the patency duration of this native bypassed graft take? What about its behavior and course? Which one is the best, to do an operation and place a graft or to wait and observe the duration of native bypass graft course? Because, as well-known, if an unnecessary graft is placed in the presence of a working vessel, the competition between them develops and both the vessel and the graft become clogged. So, when confronted an angiographic view as in the figures of this article, what is the most suitable time for surgical planning? In order to give right answers to these questions above, we need a new and special predictor indicating of the angiogenic power of the limb and its maintainability. Ankle-brachial index, colored Doppler ultrasonographic blood flow patterns and other current evaluation methods are presenting just the recent situations of

*Correspondence to: Ilker Alat, MD, Associate Professor, Department of Cardiovascular Surgery, Afyonkarahisar State Hospital, Afyonkarahisar, Turkey, E-mail: ilkeralat@hotmail.com

Key words: angionesis, bypass, predictor, graft, surgery, limb ischemia

Received: June 15, 2018; Accepted: June 21, 2018; Published: June 23, 2018 


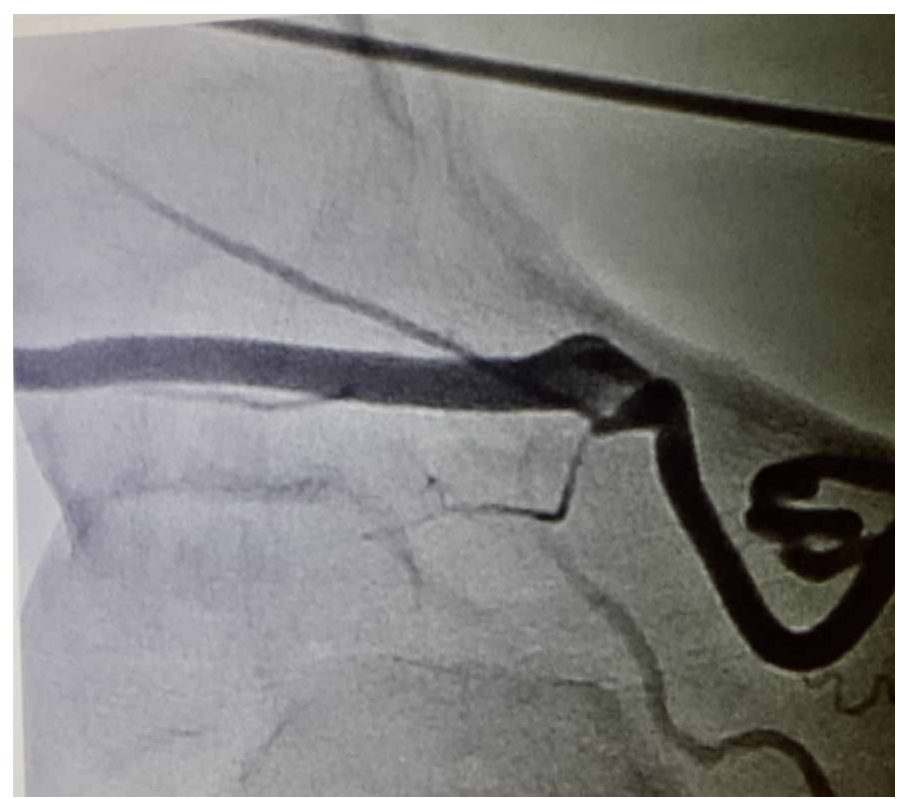

Figure 2. The angiographic view of the same collateral artery: Please note that it is like a if natively being anastomosed to the popliteal artery of the patient and also seems almost in the same caliber with it

the patients and unfortunately, they do not give any information about the power of the angiogenic capacity of the limb or maintainability of the native bypassed graft developed due to increased hypoxia in the limb. So, we need a predictor for evaluating the angiogenic capacity of the critically ischemic limbs.

\section{This predictor should be}

1. applied easily as evaluating tissue oxygenation with pulse-oximeter

2. specific and sensitive to angiogenic markers

3. able to work easily inside of the affected limb (e.g. by sinking a special designed needle into the muscle of the leg)

4. tested as soon as applied like a glucometer
5. and also, it should give an absolute information about the angiogenic capacity of the limb and the behavior and course of the existed native bypass graft.

If we can find a predictor like that, it's obvious that all of the guidelines for the patients with critical limb ischemia will re-write. And it's clear that we all, the surgeons and patients extremely need a predictor like that to make a rightest decision for bypass surgeries.

\section{Conclusion}

In conclusion, it is believed that due to this viewpoint and ideas contained in this article, this manuscript will prepare and trigger the baseline for many further works to be done in this regard.

\section{Conflict of interest}

The author of this manuscript declares that he has no conflict of interest with any company or group.

\section{References}

1. Tunis SR, Bass EB, Steinberg EP (1991) The use of angioplasty, bypass surgery, and amputation in the management of peripheral vascular disease. N Engl J Med 325: 556-62.

2. Besnier M, Gasparino S, Vono R, Sangalli E, Facoetti A, Bollati V, et al. (2018) Mir-210 enhances the therapeutic potential of bone-marrow-derived circulating proangiogenic cells in the setting of limb ischemia. Mol Ther 14. pii: S1525-0016(18)30262-4. doi: 10.1016/j.ymthe.2018.06.003. [Epub ahead of print]

3. Lian W, Hu X, Pan L, Han S, Cao C, Jia Z, et al. (2018) Human primary CD34 $4^{+}$cells transplantation for critical limb ischemia. J Clin Lab Anal 11: e22569. doi: 10.1002/ jcla.22569. [Epub ahead of print].

4. Berndt R, Hummitzsch L, Heß K, Albrecht M, Zitta K, Rusch R, et al. (2018) Allogeneic transplantation of programmable cells of monocytic origin (PCMO) improves angiogenesis and tissue recovery in critical limb ischemia (CLI): a translational approach. Stem Cell Res Ther 9: 117.

5. Uhl C, Grosch C, Hock C, Topel I, Steinbauer M (2017) Comparison of long-term outcomes of heparin bonded polytetrafluoroethylene and autologous vein below knee femoropopliteal bypasses in patients with critical limb ischaemia. Eur J Vasc Endovasc Surg 54: 203-211.

6. Johnston LE, Tracci MC, Kern JA, Cherry KJ, Kron IL, Upchurch GR Jr, et al. (2017) Surgeon, not institution, case volume is associated with limb outcomes after lower extremity bypass for critical limb ischemia in the Vascular Quality Initiative. $J$ Vasc Surg 66: 1457-1463.

Copyright: (C2018 Alat I. This is an open-access article distributed under the terms of the Creative Commons Attribution License, which permits unrestricted use, distribution, and reproduction in any medium, provided the original author and source are credited. 